\title{
Symmetric Quadrature Formulae for Simplexes
}

\author{
By P. Silvester
}

\begin{abstract}
Symmetric interpolation polynomials are defined for $N$-dimensional simplexes with the aid of a symmetric coordinate notation. These polynomials are used to produce symmetric interpolatory quadrature formulae of arbitrary degree of precision over simplexes of arbitrary dimensionality. Tabulated values of weight coefficients are given for triangles and tetrahedra.
\end{abstract}

1. Introduction. Interpolative quadrature formulae for $N$-dimensional simplexes have been given by various authors, e.g., Stroud [1] or Hammer and Stroud [2]. Their principal attraction in applications lies in the fact that multidimensional regions of integration can often be closely approximated by unions of simplexes. The object of this paper is to show that, for any $N$, it is possible to define quadrature formulae of any degree of precision $n$, symmetric in the sense of Hammer, Marlowe, and Stroud [3]; and to give a straightforward procedure for finding the weights and node locations. Although the resulting quadrature formulae are not efficient in the sense of [3], they possess the advantage of being very convenient computationally, and can be generated easily for any reasonable values of $N$ and $n$. They represent a natural generalization of the Newton-Cotes formulae to the $N$-dimensional case, and include the latter for $N=1$.

2. Notation for Simplexes. A simplex is defined by its $N+1$ vertices in the $N$-space spanned by the coordinates $x^{(1)}, x^{(2)}, \cdots, x^{(N)}$. Let $S$ be the $N$-dimensional simplex whose $k$ th vertex coordinates are $x_{k}{ }^{(i)}, i=1,2, \cdots, N$. Let the size of the simplex $S$ be denoted by $\sigma(S)$ and defined as

$$
\sigma(S)=\frac{1}{N !}\left|\begin{array}{cccc}
1 & x_{1}{ }^{(1)} & \cdots & x_{1}{ }^{(N)} \\
& \ldots & \ldots & \cdots \\
1 & x_{N+1}^{(1)} & \cdots & x_{N+1}^{(N)}
\end{array}\right| .
$$

Under this definition, the size of a 1-simplex is its length, that of a 2-simplex its area, and so on.

Let a point $P$ be located within the simplex $S$. Let $S_{m}$ denote the simplex defined by $P$ and the vertices of $S$ other than the $m$ th, i.e., by vertices $1,2, \cdots$, $m-1, m+1, \cdots, N, N+1$ of $S$, and the point $P . S_{m}$ is contained entirely within $S$. Any interior point $P$ thus defines a unique partitioning of $S$ into $N+1$ subsimplexes $S_{k}, k=1,2, \cdots, N+1$. Using Eq. (1), it is readily verified that

$$
\sum_{k=1}^{N+1} \sigma\left(S_{k}\right)=\sigma(S) \text {. }
$$

Received April 23, 1969.

AMS Subject Classifications. Primary 6555; Secondary 6520, 6505.

Key Words and Phrases. Quadrature formulae, interpolation polynomials, Newton-Cotes quadrature, multivariate interpolation, multivariate quadrature. 
Let

$$
\zeta_{m}=\sigma\left(S_{m}\right) / \sigma(S)
$$

These numbers may be termed simplex coordinates, alluding to the fact that $S$ forms the portion $0 \leqq \zeta_{m} \leqq 1, m=1, \cdots, N+1$, of the hyperplane

$$
\sum_{m=1}^{N+1} \zeta_{m}=1
$$

in the Cartesian $N+1$-space spanned by $\zeta_{1}, \zeta_{2}, \cdots, \zeta_{N+1}$, by virtue of (2). Geometrically, the simplex coordinate $\zeta_{m}$ measures normalized distance toward the $m$ th vertex orthogonally from the $N-1$-dimensional simplex defined by all vertices of $S$ other than the $m$ th.

3. Interpolation Polynomials. Let polynomials $R_{m}(z)$ be defined by

$$
\begin{aligned}
& R_{m}(z)=\prod_{k=0}^{m-1} \frac{z-z_{k}}{z_{m}-z_{k}}, \quad m>0 \\
& R_{0}(z)=1,
\end{aligned}
$$

where the $z_{k}$ are arbitrary distinct numbers, $0 \leqq z_{k} \leqq 1$. An interpolation polynomial in 1-space, of degree $n$, is then given by

$$
\alpha_{i j}=R_{i}\left(\zeta_{1}\right) R_{j}\left(\zeta_{2}\right), \quad i+j=n
$$

provided the numbers $z_{k}$ are chosen symmetrically, that is, provided that

$$
z_{p}+z_{q}=1 \text { whenever } p+q=n \text {. }
$$

Choosing $z_{k}=k / n$, the polynomials $\alpha_{i j}$ turn out to be the conventional closed Newton-Cotes interpolation polynomials, while the choice $z_{k}=(k+1) /(n+2)$ produces the open Newton-Cotes polynomials, expressed in terms of $\zeta_{1}$ and $\zeta_{2}$.

Equations (6) and (7) suggest a direct generalization for the $N$-dimensional case. Let

$$
\alpha_{k_{1} k_{2} \cdots k_{N+1}}=: \prod_{i=1}^{N+1} R_{k_{i}}\left(\zeta_{i}\right), \quad \sum_{i=1}^{N+1} k_{i}=n
$$

subject to the proviso

$$
\sum_{i=1}^{N+1} z_{l_{i}}=1 \quad \text { whenever } \sum_{i=1}^{N+1} l_{i}=n .
$$

$\Lambda$ solution, valid for any $N$ and $n$, of (9) is

$$
z_{k}=\frac{k+\mu}{n+\mu(N+1)}
$$

where $\mu$ is a nonnegative parameter. The specific choices $\mu=0$ and $\mu=1$ lead to equispaced point lattices over the simplex, with the boundary nodes included and not included, respectively. When $N=1$, these correspond to the closed and open Newton-Cotes interpolation nodes. In the closed case $\mu=0$ the auxiliary polynomials $R_{m}(z)$ become 


$$
R_{m}(z)=\frac{1}{m !} \prod_{k=0}^{m-1}(N z-k), \quad m>0
$$

and in the open case, $\mu=1$, there is obtained

$$
R_{m}(z)=\frac{1}{m !} \prod_{k=1}^{m}[(n+N+1) z-k], \quad m>0 .
$$

Clearly, other polynomials and corresponding node placements will result from other choices of $\mu$.

4. Quadrature Formulae. In general, all interpolatory quadrature formulae may be written in the form [4]

$$
\int_{S} f\left(x^{(1)}, \cdots\right) d U \simeq \sigma(S) \sum_{i} c_{i} f_{i}
$$

where the summation extends over all the interpolation nodes in $S$. The weights $c_{i}$ are given by

$$
c_{i}=\frac{1}{\sigma(S)} \int_{S} \alpha_{i}\left(\zeta_{1}, \cdots, \zeta_{N+1}\right) d U
$$

where $\alpha_{i}$ is the polynomial associated with the $i$ th interpolation node. The integrals of monomials over a simplex are well known [5],

$$
\int \zeta_{1}{ }_{1}{ }^{1} \cdots \zeta^{p_{N+1}} d U=\frac{p_{1} ! p_{2} ! \cdots p_{N+1} ! N !}{\left(p_{1}+\cdots+p_{N+1}+N\right) !} \sigma(S) .
$$

The quadrature weights $c_{i}$ are therefore readily evaluated by expanding the polynomials and integrating term by term. All required arithmetic steps are direct, and can be coded for evaluation by computer. There is no essential difficulty in obtaining formulae for any reasonable $N$ and $n$.

5. Quadrature Weights for $N=2$ and $N=3$. For $N=1$, (14) gives the wellknown and extensively tabulated Newton-Cotes quadrature weights for onedimensional integration. For $N=2$ and $N=3$, corresponding tabulations appear below. To economize on the extent of the tables, the weights have been numbered with $N+1$ subscripts corresponding to those of the interpolation polynomials; e.g., for $N=2$,

$$
c_{i j k}=\frac{1}{\sigma(S)} \int \alpha_{i j k}\left(\zeta_{1}, \zeta_{2}, \zeta_{3}\right) d U .
$$

From (8) and (15), it is readily seen that the formulae are symmetric, and the weights the same for any permutation of $i, j, k$ :

$$
c_{i j k}=c_{i k j}=c_{k i j}=\cdots .
$$

Only one of these weights needs to be tabulated. In the tables, the weights are expressed exactly as integer quotients, the numerators being tabulated individually and one common denominator being shown for each of the open and closed forms.

Application of the tabulated data to a specific simplex is made as follows. Let the vertices of the triangle (or tetrahedron) be $X_{0}, X_{1}, X_{2}$ (or $X_{0}, X_{1}, X_{2}, X_{3}$ ), 
P. SILVESTER

TABLE I

Newton-Cotes Quadrature Weights for Triangles

\begin{tabular}{|c|c|c|c|c|c|c|}
\hline \multirow{3}{*}{$\begin{array}{c}\text { Degree } \\
n\end{array}$} & \multirow{3}{*}{$\begin{array}{l}\text { Point } \\
\text { index }\end{array}$} & \multicolumn{4}{|c|}{$W E I G H T S$} & \multirow{3}{*}{$\begin{array}{c}\text { Number of } \\
\text { points } \nu\end{array}$} \\
\hline & & \multicolumn{2}{|c|}{ Numerators } & \multicolumn{2}{|c|}{ Denominators } & \\
\hline & & closed & open & & open & \\
\hline 1 & 100 & 1 & 1 & 3 & 3 & 3 \\
\hline 2 & $\begin{array}{l}200 \\
110\end{array}$ & $\begin{array}{l}0 \\
1\end{array}$ & $\begin{array}{r}7 \\
-3\end{array}$ & 3 & 12 & $\begin{array}{l}3 \\
3\end{array}$ \\
\hline 3 & $\begin{array}{l}300 \\
210 \\
111\end{array}$ & $\begin{array}{r}4 \\
9 \\
54\end{array}$ & $\begin{array}{r}8 \\
3 \\
-12\end{array}$ & 120 & 30 & $\begin{array}{l}3 \\
6 \\
1\end{array}$ \\
\hline 4 & $\begin{array}{l}400 \\
310 \\
220 \\
211\end{array}$ & $\begin{array}{r}0 \\
4 \\
-1 \\
8\end{array}$ & $\begin{array}{r}307 \\
-316 \\
629 \\
-64\end{array}$ & 45 & 720 & $\begin{array}{l}3 \\
6 \\
3 \\
3\end{array}$ \\
\hline 5 & $\begin{array}{l}500 \\
410 \\
320 \\
311 \\
221\end{array}$ & $\begin{array}{r}11 \\
25 \\
25 \\
200 \\
25\end{array}$ & $\begin{array}{r}71 \\
-13 \\
57 \\
-167 \\
113\end{array}$ & 1008 & 315 & $\begin{array}{l}3 \\
6 \\
6 \\
3 \\
3\end{array}$ \\
\hline 6 & $\begin{array}{l}600 \\
510 \\
420 \\
411 \\
330 \\
321 \\
222\end{array}$ & $\begin{array}{r}0 \\
36 \\
-27 \\
72 \\
64 \\
72 \\
-54\end{array}$ & $\begin{array}{r}767 \\
-1257 \\
2901 \\
387 \\
-3035 \\
-915 \\
3509\end{array}$ & 840 & 2240 & $\begin{array}{l}3 \\
6 \\
6 \\
3 \\
3 \\
6 \\
1\end{array}$ \\
\hline 7 & $\begin{array}{l}700 \\
610 \\
520 \\
511 \\
430 \\
421 \\
331 \\
322\end{array}$ & $\begin{array}{r}1336 \\
2989 \\
3577 \\
32242 \\
2695 \\
-6860 \\
44590 \\
3430\end{array}$ & $\begin{array}{r}898 \\
-662 \\
1573 \\
-2522 \\
-191 \\
2989 \\
-5726 \\
1444\end{array}$ & 259200 & 4536 & $\begin{array}{l}3 \\
6 \\
6 \\
3 \\
6 \\
6 \\
3 \\
3\end{array}$ \\
\hline 8 & $\begin{array}{l}800 \\
710 \\
620 \\
611 \\
530 \\
521 \\
440 \\
431 \\
422 \\
332\end{array}$ & $\begin{array}{r}0 \\
368 \\
-468 \\
704 \\
1136 \\
832 \\
-1083 \\
672 \\
-1448 \\
1472\end{array}$ & $\begin{array}{r}1051445 \\
-2366706 \\
6493915 \\
1818134 \\
-9986439 \\
3757007 \\
12368047 \\
478257 \\
10685542 \\
-6437608\end{array}$ & 14175 & 3628800 & $\begin{array}{l}3 \\
6 \\
6 \\
3 \\
6 \\
6 \\
3 \\
6 \\
3 \\
3\end{array}$ \\
\hline
\end{tabular}

where $X_{i}$ denotes a two (or three) component vector. For a quadrature formula of precision $n$, each node is designated by three (or four) integers $z_{i}$ such that $\sum z_{i}=n$; these nodes will be located at the points $\sum_{i=0}^{2}\left(z_{i}+\mu\right) X_{i} /(n+3 \mu)$ on the triangle, 
or the points $\sum_{i=0}^{3}\left(z_{i}+\mu\right) X_{i} /(n+4 \mu)$ in the tetrahedron. Formulae of open type are obtained by taking $\mu=1$, of closed type by taking $\mu=0$. Each of the $\nu$ distinct points obtained by permuting the point indices $z_{i}$ is to be assigned a weight $N(\mu) \sigma / D(\mu), \sigma$ being the area (or volume) of the simplex, $N(\mu)$ and $D(\mu)$ the tabulated numerator and denominator. As an example of this procedure, the node index numbers and quadrature weights are given in Fig. 1 for the closed formula with $n=3, N=2$.

The tabulated figures have been verified by computing integrals of monomials, and some polynomials, up to and including degree $n+2$. Their degree of precision has been verified as $n$.

TABLE II

Newton-Cotes Quadrature Weights for Tetrahedra

\begin{tabular}{|c|c|c|c|c|c|c|}
\hline \multirow{3}{*}{$\begin{array}{c}\text { Degree } \\
n\end{array}$} & \multirow{3}{*}{$\begin{array}{l}\text { Point } \\
\text { index }\end{array}$} & \multicolumn{4}{|c|}{$W E I G H T S$} & \multirow{3}{*}{$\begin{array}{l}\text { Number } 0 \\
\text { points } \nu\end{array}$} \\
\hline & & \multicolumn{2}{|c|}{ Numerators } & \multicolumn{2}{|c|}{ Denominators } & \\
\hline & & closed & open & closed & open & \\
\hline 1 & 1000 & 1 & 1 & 4 & 4 & 4 \\
\hline 2 & 2000 & -1 & 11 & & & 4 \\
\hline & 1100 & 4 & -4 & 20 & 20 & 6 \\
\hline 3 & 3000 & 1 & 20 & & & 4 \\
\hline & 2100 & 0 & 13 & 40 & 120 & 12 \\
\hline & 1110 & 9 & -29 & & & 4 \\
\hline 4 & 4000 & -5 & 79 & & & 4 \\
\hline & 3100 & 16 & -68 & & & 12 \\
\hline & 2200 & -12 & 142 & 420 & 210 & 6 \\
\hline & 2110 & 16 & -12 & & & 12 \\
\hline & 1111 & 128 & 2 & & & 1 \\
\hline 5 & 5000 & 33 & 277 & & & 4 \\
\hline & 4100 & -35 & 97 & & & 12 \\
\hline & 3200 & 35 & 223 & 4032 & 2240 & 12 \\
\hline & 3110 & 275 & -713 & & & 12 \\
\hline & 2210 & -75 & 505 & & & 12 \\
\hline & 2111 & 375 & -53 & & & 4 \\
\hline 6 & 6000 & -7 & 430 & & & 4 \\
\hline & 5100 & 24 & -587 & & & 12 \\
\hline & 4200 & -30 & 1327 & & & 12 \\
\hline & 4110 & 0 & 187 & & & 12 \\
\hline & 3300 & 40 & -1298 & 1400 & 1512 & 6 \\
\hline & 3210 & 30 & -398 & & & 24 \\
\hline & 3111 & 180 & 22 & & & 4 \\
\hline & 2220 & -45 & 1537 & & & 4 \\
\hline & 2211 & 0 & -38 & & & 6 \\
\hline
\end{tabular}

6. Conclusion. The Newton-Cotes quadrature formulae, well known for onedimensional integration, lend themselves to direct generalisation in $N$-dimensional simplexes. For the most important two- and three-dimensional cases, weight co- 
efficients for open and closed forms have been computed and tabulated.

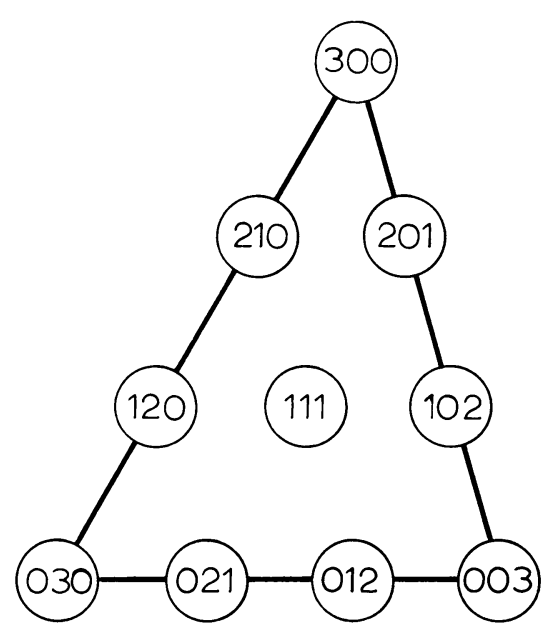

a

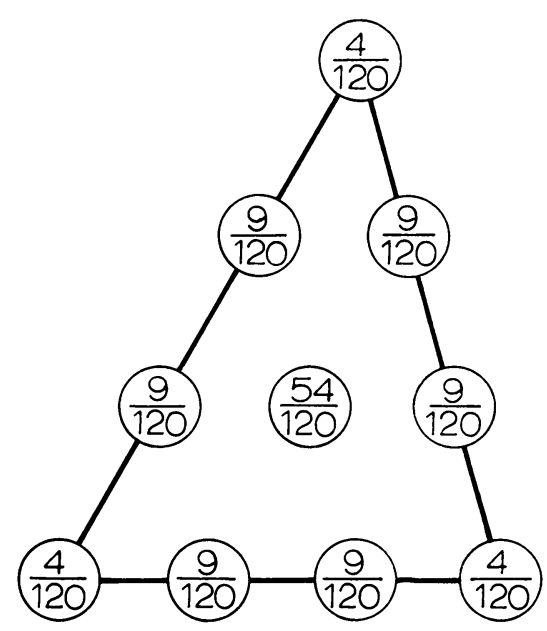

$b$

FIGURe 1. Cubic Quadrature over a Triangle. (a) Index Numbering of Quadrature Nodes, (b) Quadrature Weights at the Nodes.

The author wishes to acknowledge financial support of the above work by the National Research Council of Canada, and the Defence Research Board of Canada.

\section{McGill University}

Montreal 110, P.Q.

Canada

1. A. H. Stroud, "Approximate integration formulas of degree 3 for simplexes," Math. Comp., v. 18,1964 , pp. 590-597. MR $29 \# 6628$.

2. P. C. HAMmer \& A. H. STRoud, "Numerical integration over simplexes," $M T A C$, v. 10, 1956, pp. 137-139. MR 19, 177.

3. P. C. Hammer, O. J. Marlowe \& A. H. Stroud, "Numerical integration over simplexes and cones," MTAC, v. 10, 1956, pp. 130-137. MR 19, 177.

4. E. IsaAcson \& H. B. KELLER, Analysis of Numerical Methods, Wiley, New York, 1966. MR 34 \#924.

5. G. C. BEst, "Helpful formulas for integrating polynomials in three dimensions," Math. Comp., v. 18, 1964, pp. 310-312. MR 32 \#3261. 